\title{
Trends \& Treatments of Corona -A Pandemic Disaster
}

\author{
Anamika $\mathrm{PK}^{1^{*}}$, Dr. P. Muralidharan ${ }^{2}$, Sandhiya $\mathrm{V}^{3}$, E. Sankari ${ }^{4}$
}

${ }_{1}^{1}$ Assistant Professor, Department of Pharmacology, C.L. Baid Metha College of Pharmacy Chennai-97 India
${ }^{2}$ Professor and HOD Department of Pharmacology, C.L. Baid Metha College of Pharmacy Chennai-97 India
${ }^{3}$ Assistant Professor, Department of Pharmaceutics, C.L. Baid Metha College of Pharmacy Chennai-97 India
${ }^{4}$ Assistant Professor, Department of Pharmaceutical analysis, C.L. Baid Metha College of Pharmacy, Thoraipakkam, Chennai-97 India

DOI: $10.36347 /$ sajp.2020.v09i04.001

| Received: 25.03.2020 | Accepted: 02.04.2020 | Published: 05.04.2020

*Corresponding author: Anamika PK

Coronaviruses are zoonotic, meaning they are transmitted between animals and people. It is a group of highly diverse single stranded RNA virus. Corona included fever fatigue cough cold Coronaviruses (CoV) are immeasurable family of viruses that cause sickness ranging from the common cold to more severe viruses such as Middle East Respiratory Syndrome (MERS-CoV) and Severe Acute Respiratory Syndrome (SARS-CoV). 2-14 days represents the current official estimated range for the novel coronavirus COVID-19. The World Health Organization (WHO) declared the 2019-20 coronavirus outbreak a pandemic and a Public Health Emergency of International Concern (PHEIC).

Keywords: Coronaviruses (CoVID-19), Infection, Pandemic.

Copyright @ 2020: This is an open-access article distributed under the terms of the Creative Commons Attribution license which permits unrestricted use, distribution, and reproduction in any medium for non-commercial use (NonCommercial, or CC-BY-NC) provided the original author and source are credited.

\section{INTRODUCTION}

Coronavirus disease (COVID-19) is a new strain that was discovered in 2019 and has not been formerly acknowledged in humans. The $\mathrm{S}$ protein envelope spike is important for corona virus. The $\mathrm{S}$ protein mediates the Receptor Protein and Membrane Fusion crucial for determining host capacity and transmission. This S-Protein is functionally divided into two domains $\mathrm{S} 1$ and S2.S1 domain is responsible for receptor binding and $\mathrm{S} 2$ domain is responsible for Cell membrane fusion.

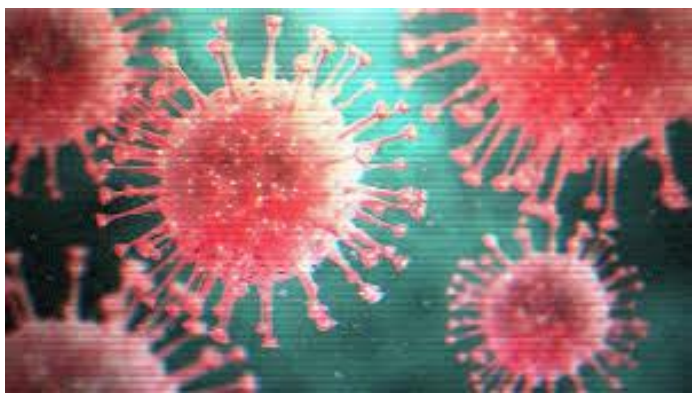

Thorough investigations found that SARS$\mathrm{CoV}$ was transmitted from civet cats to humans and MERS-CoV from dromedary camels to Human beings. Several known coronaviruses are socializing in animals that have not yet infected humans.

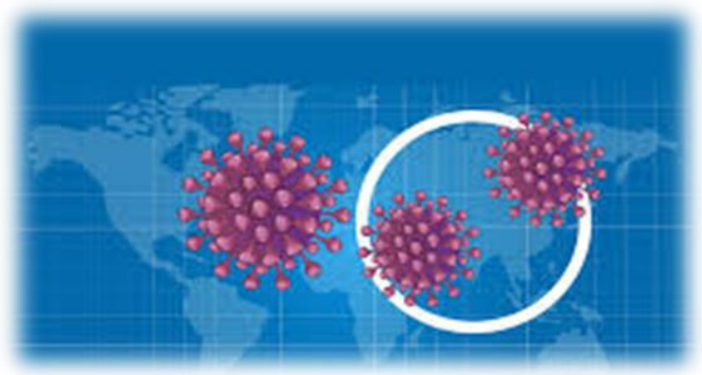

Common signs of contamination include respirational symptoms, temperature, and cough, shortness of breath and breathing problems. In more severe cases, infection can cause pneumonia, severe acute respiratory syndrome, kidney failure and even death.

Standard recommendations to stop contamination blowout consist of regular hand washing, masking the mouth and nose once coughing and sneezing, thoroughly cooking meat and eggs. Avoid close connection with anybody showing symptoms of respiratory complaint such as coughing and sneezing.

Coronavirus disease spreads primarily through contact with an infected person when they cough or sneeze. It also spreads when a person touches a surface or object that has the virus on it, then touches their eyes, nose, or mouth. 


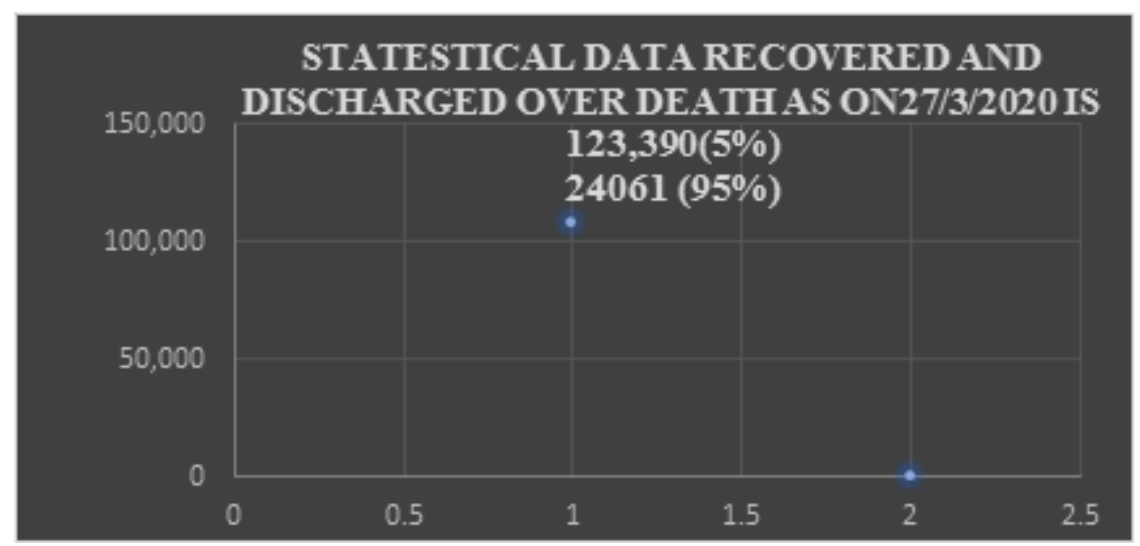

Incubation Period: 2 - 14 days

\section{COVID-19 Incubation Period}

The incubation period (time from exposure towards the development of symptoms) of the virus is estimated to be between 2 and 14 days based on the following sources:

- The World Health Organization (WHO) reported an incubation period for COVID-19 between 2 and 10 days.

- China's National Health Commission (NHC) had initially estimated an incubation period from 10 to 14 days

- The United States' CDC estimates the incubation period for COVID-19 to be between 2 and 14 days.

- DXY.cn, a leading Chinese online community for physicians and health care professionals, is reporting an incubation period of "3 to 7 days, up to 14 days".

The incubation period has been found to be as long as 24 days (range: 0-24 days; median: 3.0 days) in a study published on February 9.

The WHO said a very long incubation period could reflect a double exposure. 24 days represented an outlier observation that must be taken into consideration in the context of the main finding of the study. WHO is not considering changing recommendations regarding incubation periods?

More recently, however, a case with an incubation period of 19 days was observed in a JAMA study published on Feb. 21, and another case with an incubation period of 27 days was reported by Hubei Province on Feb. 22. Incubation period of 5.2 days on average

A Chinese study published in the New England Journal of Medicine on Jan. 30[7], has found the incubation period to be 5.2 days on average, but it varies greatly among patients. The Chinese team conducting the study said their findings support a 14day medical observation period for people exposed to the pathogen.

\section{Incubation Period in Travelers from Wuhan}

A study financed by the Netherlands Ministry of Health and published on Euro surveillance, analyzed data on 88 cases with known travel history (to and) from Wuhan which were detected between 20 and 28 January as being infected with COVID-19.

The mean incubation period was estimated to be 6.4 days. The incubation period ranges from 2.1 to 11.1 days. The upper limit of 11.1 days could be considered conservative.

\section{The importance of knowing the incubation period}

Understanding the incubation period is very important for health authorities as it allows them to introduce more effective quarantine systems for people suspected of carrying the virus, as a way of controlling and hopefully preventing the spread of the virus.

\section{Comparison with other viruses}

For comparison, the incubation period for the common flu (seasonal influenza) is typically around 2 days. Incubation period for other coronaviruses: SARS 2-7 days; MERS 5 days typically (range 2-14 days).

\begin{tabular}{|c|c|}
\hline VIRUS & $\begin{array}{l}\text { INCUBATION PERIOD } \\
\text { (TYPICAL CASES) }\end{array}$ \\
\hline $\begin{array}{l}\text { Novel Coronavirus } \\
\text { (COVID-19) }\end{array}$ & $2-14$ or $0-24$ days $*$ \\
\hline SARS & $\begin{array}{l}\text { 2-7 days, } \\
\text { as long as } 10 \text { days }\end{array}$ \\
\hline MERS & 5 days (range: $2-14$ ) \\
\hline Swine Flu & $\begin{array}{l}1-4 \text { days, } \\
\text { as long as } 7 \text { days }\end{array}$ \\
\hline Seasonal Flu & 2 days (1-4 range) \\
\hline Virus & $\begin{array}{l}\text { Incubation Period } \\
\text { (typical cases) }\end{array}$ \\
\hline $\begin{array}{l}\text { Novel Coronavirus } \\
\text { (COVID-19) }\end{array}$ & $2-14$ or $0-24$ days \\
\hline
\end{tabular}

During the past 3 weeks, new major epidemic foci of coronavirus disease 2019 (COVID-19), some without traceable origin, have been identified and are rapidly expanding in Europe, North America, Asia, and 
the Middle East, with the first confirmed cases being identified in African and Latin American countries. By March 16, 2020, the number of cases of COVID-19 outside China had increased drastically and the number of affected countries, states, or territories reporting infections to WHO was 143.

On the basis of" alarming levels of spread and severity and by the alarming levels of inaction", on March 11, 2020, the Director-General of WHO characterized the COVID-19 situation as a pandemic.

The WHO Strategic and Technical Advisory Group for Infectious Hazards (STAG-IH) regularly reviews and updates its risk assessment of COVID-19 to make recommendations to the WHO health emergencies programme. STAG-IH's most recent formal meeting on March 12, 2020, included an update of the global COVID-19 situation and an overview of the research priorities established by the WHO Research and Development Blueprint Scientific Advisory Group that met on March 2, 2020, in Geneva, Switzerland, to prioritise the recommendations of an earlier meeting on COVID-19 research held in early February, 2020.

In this Comment, we outline STAG-IH's understanding of control activities with the group's risk assessment and recommendations. To respond to COVID-19, many countries are using a combination of containment and mitigation activities with the intention of delaying major surges of patients and levelling the demand for hospital beds, while protecting the most vulnerable from infection, including elderly people and those with comorbidities. Activities to accomplish these goals vary and are based on national risk assessments that many times include estimated numbers of patients requiring hospitalization and availability of hospital beds and ventilation support. Most national response strategies include varying levels of contact tracing and self-isolation or quarantine; promotion of public health measures, including handwashing, respiratory etiquette, and social distancing; preparation of health systems for a surge of severely ill patients who require isolation, oxygen, and mechanical ventilation; strengthening health facility infection prevention and control, with special attention to nursing home facilities; and postponement or cancellation of large-scale public gatherings.

Some lower-income and middle-income countries require technical and financial support to successfully respond to COVID-19, and many African, Asian, and Latin American nations are rapidly developing the capacity for PCR testing for COVID-19.

Based on more than 500 genetic sequences submitted to GISAID (the Global Initiative on Sharing All Influenza Data), the virus has not drifted to significant strain difference and changes in sequence are minimal. There is no evidence to link sequence information with transmissibility or virulence of severe acute respiratory syndrome coronavirus 2 (SARS-CoV2), the virus that causes COVID-19.

SARS-CoV-2, like other emerging high-threat pathogens, has infected health-care workers in China and several other countries. To date, however, in China, where infection prevention and control was taken seriously, nosocomial transmission has not been a major amplifier of transmission in this epidemic. Epidemiological records in China suggest that up to $85 \%$ of human-to-human transmission has occurred in family clusters and that 2055 health-care workers have become infected, with an absence of major nosocomial outbreaks and some supporting evidence that some health-care workers acquired infection in their families.

These findings suggest that close and unprotected exposure is required for transmission by direct contact or by contact with fomites in the immediate environment of those with infection. Continuing reports from outside China suggest the same means of transmission to close contacts and persons who attended the same social events or were in circumscribed areas such as office spaces or cruise ships.

Intensified case finding and contact tracing are considered crucial by most countries and are being undertaken to attempt to locate cases and to stop onward transmission. Confirmation of infection at present consists of PCR for acute infection, and although many serological tests to identify antibodies are being developed, they require validation with well characterized sera before they are reliable for general use.

From studies of viral shedding in patients with mild and more severe infections, shedding seems to be greatest during the early phase of disease (Myoung-don Oh and Gabriel Leung, WHO Collaborating Centre for Infectious Disease Epidemiology and Control, School of Public Health, LKS Faculty of Medicine, The University of Hong Kong, Hong Kong, Special Administrative Region, China, personal communication).

The role, if any, of asymptomatic carriers in transmitting infection is not yet completely understood. Presymptomatic infectiousness is a concern (Myoung-don $\mathrm{Oh}$ and Gabriel Leung, personal communication) and many countries are now using 1-2 days of symptom onset as the start day for contact identification.

A comprehensive report published by the Chinese Center for Disease Control and Prevention on the epidemiological characteristics of 72314 patients with COVID-19 confirmed previous understanding that 
most known infections cause mild disease, with a case fatality ratio that ranged from $2.9 \%$ in Hubei province to $0.4 \%$ in the other Chinese provinces.

This report also suggested that elderly people, particularly those older than 80 years, and people with comorbidities, such as cardiac disease, respiratory disease, and diabetes, are at greatest risk of serious disease and death. The case definition used in China changed several times as COVID-19 progressed, making it difficult to completely characterize the natural history of infection, including the mortality ratio.

Information on mortality and contributing factors from outbreak sites in other countries varies greatly, and seems to be influenced by such factors as age of patients, associated comorbidities, and availability of isolation facilities for acute care for patients who need respiratory support, and surge capacity of the health-care system. Individuals in care facilities for older people are at particular risk of serious disease as shown in the report of a series of deaths in an elderly care facility in the USA.

The pandemic of COVID-19 has clearly entered a new stage with rapid spread in countries outside China and all members of society must understand and practice measures for self-protection and for prevention of transmission of infection to others. STAG-IH makes the following recommendations.

First, countries need to rapidly and robustly increase their preparedness, readiness, and response actions based on their national risk assessment and the four WHO transmission scenarios for countries with no cases, first cases, first clusters, and community transmission and spread (4Cs).

Second, all countries should consider a combination of response measures: case and contact finding; containment or other measures that aim to delay the onset of patient surges where feasible; and measures such as public awareness, promotion of personal protective hygiene, preparation of health systems for a surge of severely ill patients, stronger infection prevention and control in health facilities, nursing homes, and long-term care facilities, and postponement or cancellation of large-scale public gatherings.

Third, countries with no or a few first cases of COVID-19 should consider active surveillance for timely case finding; isolate, test, and trace every contact in containment; practice social distancing; and ready their health-care systems and populations for spread of infection.
Fourth, lower-income and middle-income countries that request support from WHO should be fully supported technically and financially. Financial support should be sought by countries and by WHO, including from the World Bank Pandemic Emergency Financing Facility and other mechanisms.

Finally, research gaps about COVID-19 should be addressed and are shown in the accompanying board and include some identified by the global community and by the Research and Development Blueprint Scientific Advisory Group.

How is the coronavirus spreading around the world?

The coronavirus emerged in Wuhan, a city of 11 million people in China's Hubei province, in late 2019. Cases of the disease it causes, COVID-19, grew by several thousand per day in China in late January and early February, the peak of the epidemic there. The number of infections appearing each day has since plummeted in China, owing in large part to containment efforts, but the outbreak is now a global pandemic. Large outbreaks in South Korea, Iran, Italy and elsewhere have propelled a spike in international cases across more than 150 countries. The SARS coronavirus was found to have jumped to people from civet cats that had picked it up from bats. The COVID-19 virus, called SARS-CoV-2, is also thought to have come from bats, either directly or through an as-yet unidentified mammal. Both viruses caused chaos and economic disaster. But the two outbreaks have progressed very differently, especially in the speed and extent of spread.

The mysterious coronavirus outbreak in the Chinese city Wuhan, now termed as COVID-19, and its fast spread to many other countries, endangers thousands of lives. The pandemic has catalyzed the development of novel coronavirus vaccines across the biotech industry, both by pharmaceutical companies and research organizations such as the National Institutes of Health (NIH), US.The first COVID-19 vaccine in China is expected to be ready for clinical trials by the end of April, according to $\mathrm{Xu}$ Nanping, China's vice-minister of science and technology. Inovio Pharmaceuticals plans to begin clinical trials on a coronavirus vaccine in April this year. Health officials from WHO have noted that Gilead's remdesivir has demonstrated efficacy in treating the coronavirus infection.

\section{Chloroquine to be tested for coronavirus treatment in the US}

The President of the United States, Donald Trump, announced on 19 March that chloroquine (hydroxychloroquine/Plaquenil), a drug used to treat malaria and arthritis, was approved by the US Food and Drug Administration (FDA) to be tested as a treatment for COVID-19. Chloroquine is being tested in various clinical trials conducted by government agencies and 
academic institutions. Other antivirals drugs are also planned to be fast-tracked for testing for coronavirus.

\section{Favilavir, the first approved coronavirus drug in China}

The National Medical Products Administration of China has approved the use of Favilavir, an anti-viral drug, as a treatment for coronavirus. The drug has reportedly shown efficacy in treating the disease with minimal side effects in a clinical trial involving 70 patients. The clinical trial is being conducted in Shenzhen, Guangdong province.

\section{Pharmaceutical companies involved in developing coronavirus drugs/vaccines}

Here is a list of the major coronavirus drugs that pharmaceutical companies across the world are developing that have the potential to become major coronavirus vaccines or antivirals for treating the contagious coronavirus infection.

\section{Novel coronavirus vaccines}

Listed below are the coronavirus vaccines in various stages of development, across the world.

\section{TJM2 by I-Mab Biopharma}

I-Mab Biopharma is developed TJM2, a neutralizing antibody, as a treatment for cytokine storm in patients suffering from a severe case of coronavirus infection. The drug targets the human granulocytemacrophage colony-stimulating factor (GM-CSF), which is responsible for acute and chronic inflammation.

The company will commence development after receiving approval for the Investigational New Drug (IND) application from the U.S. Food and Drug Administration (FDA).

\section{Coronavirus vaccine by Medicago}

Medicago is developing drug candidates against COVID-19 after having produced Virus-Like Particles (VLP) of the coronavirus. The company has formed collaboration with Laval University's Infectious Disease Research Centre to develop antibodies against SARS-CoV-2.

The company's research activities are being partly funded by the Canadian Institutes for Health Research (CIHR).

\section{AT-100 by Airway Therapeutics}

Airway Therapeutics is exploring its novel human recombinant protein named AT-100 (rhSP-D) as a treatment for coronavirus. The company has announced a filing with the Respiratory Diseases Branch of the National Institutes of Health to evaluate the drug.
AT-100 has shown efficacy in preclinical studies in reducing inflammation and infection in the lungs, while also generating an immune response against various respiratory diseases.

\section{TZLS-501 by Tiziana Life Sciences}

Tiziana Life Sciences is developing its monoclonal antibody named TZLS-501 for the treatment of COVID-19. TZLS-501 is a human antiinterleukin-6 receptor (IL-6R), which helps in preventing lung damage and elevated levels of IL-6.

The drug works by binding to IL-6R and depleting the amount of IL- 6 circulating in the body thereby reducing chronic lung inflammation.

\section{OYA1 by OyaGen}

OyaGen's OYA1 has shown strong antiviral efficacy against coronavirus in laboratory essays. It was found to be more effective than chlorpromazine $\mathrm{HCl}$ in inhibiting SARS-CoV-2 from replicating in cell culture.

OYA1 was earlier approved as an investigational new drug for treating cancer but abandoned due to lack of efficacy. OyaGen plans to conduct further research on the drug to determine the efficacy in treating coronavirus.

\section{BPI-002 by Beyond Spring}

Beyond Spring's BPI-002 is a small molecule agent indicated for treating various infections including COVID-19. It has the ability to activate CD4+ helper T cells and CD8+ cytotoxic $\mathrm{T}$ cells and generating an immune response in the body.

If combined with another COVID-19 vaccine, the drug has the ability to generate long-term protection against viral infections. Beyond Spring has filed US patent protection for the drug for treating viral infections.

\section{Altimmune's intranasal coronavirus vaccine \\ An intranasal Covid-19 vaccine is being developed by US-based clinical-stage biopharmaceutical company, Altimmune's.}

Design and synthesis of the single-dose vaccine have been completed, while animal testing will follow. The coronavirus vaccine is being developed based on a vaccine technology platform that is similar to NasoVAX, an influenza vaccine developed by Altimmune's.

\section{INO-4800 by Inovio Pharmaceuticals and Beijing Advaccine Biotechnology \\ Inovio Pharmaceuticals has collaborated with} Beijing Advaccine Biotechnology Company to advance the development of the former's vaccine, INO-4800, as a novel coronavirus vaccine. The company has started pre-clinical testing for clinical product manufacturing. 
The vaccine development is supported by a $\$ 9 \mathrm{~m}$ grant from the Coalition for Epidemic Preparedness Innovations (CEPI).

Inovio announced an accelerated timeline for the development of the vaccine on 03 March. Preclinical trials are ongoing and the design for human clinical trials has been completed. The company has also prepared 3,000 doses for human clinical trials planned to be conducted across the US, China, and South Korea. Plans for large-scale manufacturing have also been developed.

Human clinical trials in 30 healthy volunteers are expected to commence in April 2020 in the US, followed by China, and South Korea. A phase one clinical trial is planned to be conducted in parallel in China, by Beijing Advaccine. Results from the clinical trials are expected to be available in September 2020.

Inovio aims to produce one million doses of the vaccine by the end of 2020 to perform additional clinical trials or emergency use.

\section{NP-120 (Ifenprodil) by Algernon Pharmaceuticals}

Algernon Pharmaceuticals has announced that it is exploring its NP-120 (Ifenprodil) as a potential treatment COVID-19. Ifenprodil is an N-methyl-daspartate (NDMA) receptor glutamate receptor antagonist sold under the brand name Cerocal. It has demonstrated efficacy in improving survivability in mice infected with H5N1.

\section{APN01 by University of British Columbia and APEIRON Biologics}

A drug candidate developed by APEIRON Biologics named APNO1 is being tested in China in a phase one pilot trial as a treatment for COVID-19. APN01 is based on research conducted by a professor at the University of British Columbia for treating SARS. The research revealed that the ACE2 protein was the main receptor for the SARS virus.

The clinical trial will test the drug's efficacy in reducing the viral load in patients. Data from the trial will be used to determine if additional clinical trials are required to be conducted in larger number of patients.

\section{mRNA-1273 vaccine by Moderna and Vaccine Research Center \\ Moderna and the Vaccine Research Center, a unit of the National Institute of Allergy and Infectious Diseases (NIAID), have collaborated to develop a vaccine for coronavirus. The vaccine targets the Spike (S) protein of the coronavirus.}

The first vials of the vaccine have been manufactured at Moderna's Massachusetts manufacturing plant and shipped to NIAID for phase one human clinical trial. The trial began on 16 March at the Kaiser Permanente Washington Health Research Institute in Seattle, Washington. A total of 45 males and females aged between 18 and 45 have been enrolled for the trial.

The participants will be divided into three cohorts who will be administered 25 microgram (mcg), $100 \mathrm{mcg}$ or $250 \mathrm{mcg}$ dose 28 days apart.

\section{Avian Coronavirus Infectious Bronchitis Virus (IBV) vaccine by MIGAL Research Institute}

The MIGAL Research Institute in Israel announced that an Infectious Bronchitis Virus (IBV) vaccine developed to treat avian coronavirus has been modified to treat COVID-19. The vaccine has demonstrated efficacy in pre-clinical trials conducted by the Volcani Institute.

The IBV vaccine was developed after four years of research and has high genetic similarity to the human coronavirus. The institute has genetically modified the vaccine to treat COVID-19 and will be available in the oral form.

The institute is currently exploring potential partners for producing the vaccine in the next eight to ten weeks and obtaining the necessary safety approvals for in-vivo testing.

\section{TNX-1800 by Tonix Pharmaceuticals}

Tonix Pharmaceuticals has partnered with Southern Research, a non-profit research organisation, to develop a coronavirus vaccine named TNX1800. The vaccine is a modified horsepox virus developed using Tonix's proprietary horsepox vaccine platform.

TNX-1800 is designed to express a protein derived from the virus that causes the coronavirus infection. Southern Research will be responsible for evaluating the efficacy of the vaccine, under the partnership.

\section{Brilacidin by Innovation Pharmaceuticals}

Innovation Pharmaceuticals announced that it is evaluating Brilacidin, a defensin mimetic drug candidate, as a potential treatment for coronavirus. Brilacidin has shown antibacterial, anti-inflammatory and immunomodulatory properties in several clinical trials.

The company is planning to explore research collaborations and seek federal grants to develop the coronavirus drug. It is already investigating the drug for inflammatory bowel disease and oral mucositis in cancer patients.

Innovation has signed two material transfer agreements with a university in the US and 12 biocontainment labs in the US for evaluation of Brilacidin as a treatment for COVID-19. One of the 
biocontainment labs is scheduled to commence testing of the drug in the third week of March.

\section{Recombinant subunit vaccine by Clover Biopharmaceuticals}

Clover Biopharmaceuticals is developing a recombinant subunit vaccine using its patented Trimer$\mathrm{Tag}^{\odot}$ technology. The company is developing the vaccine based on the trimeric $\mathrm{S}$ protein (S-Trimer) of the COVID-19 coronavirus, which is responsible for binding with the host cell and causing a viral infection.

Using $\quad$ Trimer-Tag $^{\circledR}$ technology, Clover successfully produced the subunit vaccine in a mammalian cell-culture based expression system on 10 February. The company also identified antigen-specific antibody in the serum of fully recovered patients who were previously infected by the virus.

A highly purified form of the S-Trimer vaccine is expected to be available in six to eight weeks for performing pre-clinical studies. The company is equipped with in-house cGMP biomanufacturing capabilities to scale-up production if the vaccine is proven to be successful.

Clover is also collaborating with GSK to develop a vaccine using the latter's pandemic adjuvant system.

\section{Vaxart's coronavirus vaccine}

Vaxart is developing an oral recombinant vaccine in tablet formulation using its proprietary oral vaccine platform, VAAST.

The company plans to develop vaccines based on the published genome of 2019-nCOV to be tested in pre-clinical models for mucosal and systemic immune responses.

\section{CytoDyn-leronlimab}

CytoDyn is examining leronlimab (PRO 140), a CCR5 antagonist, as a potential coronavirus drug.

The drug is already being investigated in phase two clinical trials as a treatment for HIV and has been awarded fast-track approval status by the United States Food and Drug Administration.

\section{Linear DNA Vaccine by Applied DNA Sciences and Takis Biotech \\ Applied DNA Sciences' subsidiary LineaRx and Takis Biotech formed a joint venture on 07 February to develop a linear DNA vaccine as a treatment for coronavirus. The JV will use Polymerase Chain Reaction (PCR)-based DNA manufacturing technology to develop the vaccine.}

The PCR technology offers several advantages including high purity, increased production speed, and absence of antibiotics and bacterial contaminants. Further, the vaccine gene developed through this technology can be effective without being inserted into the patient's genome.

The design for four DNA vaccine candidates is expected to be produced using the PCR technology for carrying out animal testing. The design of one of the vaccine candidates is based on the entire spike gene of the coronavirus, while the remaining is designed based on the antigenic portions of the protein.

\section{BXT-25 by BIOXYTRAN to treat late-stage acute respiratory distress syndrome (ARDS) \\ BIOXYTRAN announced that it is exploring} partners to develop its lead drug candidate, BX-25, as a treatment for Acute Respiratory Distress Syndrome (ARDS) in late-stage patients infected with the coronavirus. The diffusion of oxygen to the blood is comprised in patients suffering from ARDS leading to fluid build-up in the lungs.

BX-25 is designed to be 5,000 times smaller than blood cells and efficiently transport oxygen through the body for a period of nine hours before being processed by the liver. The drug can help in supplying oxygen to the vital organs and enable the patient to recover and survive.

\section{MERS CoV vaccines for coronavirus}

\section{Novavax's MERS coronavirus vaccine candidate}

Novavax developed a novel Middle East Respiratory Syndrome (MERS) coronavirus vaccine candidate in 2013, post the identification of the first MERS coronavirus ((MERS-CoV) in Saudi Arabia in 2012. It is a crucial target for coronavirus vaccine development by the Coalition for Epidemic Preparedness Innovations (CEPI) and is a priority disease for the World Health Organisation (WHO).

The candidate is designed to primarily bind to the major surface S-protein and developed using the company's recombinant nanoparticle vaccine technology. Tested along with the Novavax's proprietary adjuvant Matrix- $\mathrm{M}^{\mathrm{TM}}$, it inhibited infection by inducing immune responses in the laboratory studies.

Novavax has received $\$ 4 \mathrm{~m}$ in funding from CEPI to advance the development of the vaccine. The company has produced several nanoparticle vaccine candidates for testing in animal models and aims to carry out human trials in 2020.

The MERS coronavirus is related to the severe acute respiratory syndrome (SARS) coronavirus, for which the company had previously developed a recombinant nanoparticle vaccine candidate. 


\section{Inovio Pharma's INO-4700}

The investigational DNA immunotherapy, INO-4700 (GLS-5300) is being developed by Inovio in partnership with GeneOne Life Science. It is delivered as vaccine intramuscularly, using the Cellectra ${ }^{\circledR}$ delivery device.

The company has received a $\$ 5 \mathrm{~m}$ grant from the Bill and Mellinda Gates foundation to accelerate the development of the Cellectra ${ }^{\circledR}$ delivery device.

The vaccine was well-tolerated and demonstrated high immune responses against the MERS-CoV in $94 \%$ of patients in the early-stage clinical trial in July 2019.

It also generated broad-based $\mathrm{T}$ cell responses in $88 \%$ of the subjects.

"Research organisations such as the National Institutes of Health (NIH), US are also developing a vaccine for the coronavirus."

\section{Coronavirus drugs}

The novel coronavirus drugs in various stages of development globally are listed below.

\section{Remdesivir (GS-5734) by Gilead Sciences}

An ebola drug developed by Gilead Sciences that was found to be ineffective is now being tested in two phases III randomised clinical trials in Asian countries.

The trials are being performed on 761 patients in a randomised, placebo-controlled, double-blind study at multiple hospitals in Wuhan, the epicentre of the novel coronavirus outbreak. The results from the trials are expected to be available over the next few weeks.

According to a report by The New England Journal of Medicine (NEJM), remdesivir, when administered to a coronavirus patient in the US, appeared to have improved the clinical condition.

The University of Nebraska Medical Center is also carrying out clinical trials to test the safety and efficacy of the drug. The first patient to be administered the drug is an evacuee from the Diamond Princess Cruise ship.

\section{Actemra by Roche to treat coronavirus-related complications}

China approved the use of Roche's Actemra for the treatment of severe complications related to coronavirus. Drugs like Actemra have the ability to prevent cytokine storms or overreaction of the immune system, which is considered as the main reason behind organ failure leading to death in some coronavirus patients.
Actemra is also being evaluated in a clinical trial in China, which is expected to enroll 188 coronavirus patients. The clinical trial is expected to be conducted until May 10.

\section{Biocryst Pharma's Galidesivir, a potential antiviral for coronavirus treatment}

The antiviral drug Galidesivir (BCX4430) has shown broad-spectrum activity against a wide range of pathogens including coronavirus. It is a nucleoside RNA polymerase inhibitor that disrupts the process of viral replication.

The drug has already shown survival benefits in patients against deadly viruses such as Ebola, Zika, Marburg, and Yellow fever.

Galidesivir is currently in advanced development stage under the Animal Rule to combat multiple potential viral threats including coronaviruses, flaviviruses filoviruses, paramyxoviruses, togaviruses, bunyaviruses, and arenaviruses.

\section{Regeneron's REGN3048-3051 and Kevzara}

Discovered by Regeneron, the combination of neutralising monoclonal antibodies REGN3048 and REGN3051 is being studied against coronavirus infection in a first-in-human clinical trial sponsored by the National Institute of Allergy and Infectious Diseases (NIAID). The safety and tolerability of the drug will be studied in 48 patients.

Both the antibodies bind to S-protein of MERS coronavirus. The intravenous administration of the drug in the mouse model of MERS resulted in the high-level neutralisation of the MERS coronavirus in circulating blood with reduced viral loads in the lungs.

Regeneron has partnered with Sanofi to evaluated Kevzara, a fully-human monoclonal antibody, in a phase two/three clinical trial in patients with severe COVID-19 infection. Kevzara is approved for the treatment of rheumatoid arthritis and is known to block the interleukin-6 (IL-6) pathway, which causes an overactive inflammatory response in the lungs of COVID-19 patients.

\section{SNG001 by Synairgen Research}

Synairgen Research's SNG001, an inhaled drug, is planned to be tested by the University of Southampton to treat asthma, chronic obstructive pulmonary disease and lower respiratory tract illnesses caused by coronavirus.

SNG001 is a formulation of naturally occurring Interferon- $\beta$, which is administered through a nebuliser and is delivered directly to the lungs to reduce the severity of the infection caused by coronavirus. 


\section{AmnioBoost by Lattice Biologics}

Lattice Biologics is exploring the efficacy of its amniotic fluid concentrate, AmnioBoost, in treating acute respiratory distress syndrome (ARDS) in COVID19 patients. AmnioBoost was developed for chronic adult inflammatory conditions such as osteoarthritis.

The drug has shown efficacy in reducing the inflammatory conditions caused by several diseases including coronavirus. It reduces the production of proinflammatory cytokines while boosting the production of anti-inflammatory cytokines.

\section{Other companies developing coronavirus vaccines/drugs}

Companies such as Inovio Pharmaceuticals, Moderna, and Novavax have been reported to be developing coronavirus vaccines. A total of 30 therapies are being tested, including few traditional medicines for coronavirus treatment by Chinese scientists. Chloroquine phosphate has shown efficacy in treating symptoms of the disease, among the 30 therapies. Patients administered with the drug achieved a better drop in fever and shorter recovery time in clinical trials being conducted in hospitals in the Guangdong province and Hunan province.

\section{Enanta Pharmaceuticals}

Enanta Pharmaceuticals has announced its plans to develop antiviral drug candidates to treat COVID-19 patients. The company is testing compounds from its existing antiviral compound library for potential efficacy in treating COVID-19. It has also launched a drug discovery programme to develop direct-acting drug candidates to treat COVID-19.

\section{Predictive Oncology}

Predictive Oncology has launched an AI Platform for the discovery and development of vaccines against coronavirus. The company has signed an agreement with Inventa BioTech to acquire Soluble Therapeutics, which provides it with access to the HSC $^{\mathrm{TM}}$ Technology.

Predictive will use the $\mathrm{HSC}^{\mathrm{TM}}$ Technology along with its predictive modeling platform to deploy an AI discovery platform that can screen the ideal combination of additives and excipients for protein formulations.

\section{Emergent BioSolutions}

Emergent BioSolutions is developing two plasma-derived product candidates or hyperimmunes using its hyperimmune platforms for the treatment of coronavirus. The hyperimmune platforms have been used previously for the development of several approved products including vaccines for smallpox, botulism, and anthrax.
The hyperimmunes are polyclonal antibodies derived from plasma, which are capable of generating an immune response and protecting against infection. Product candidate derived from human plasma is named COVID-HIG, while COVID-EIG is derived from equine plasma. Both will be explored for the treatment of patients with a severe case of infection.

\section{Integral Molecular}

Integral Molecular has launched a vaccine programme using its two technology platforms including Shotgun Mutagenesis Epitope Mapping and the Membrane Proteome Array. The technologies will help in understanding the human immune response to the coronavirus and isolate the cellular receptors that enable the virus to spread quickly.

The Shotgun technology helps in identifying more than 1,000 binding sites for antibodies, while the Membrane Proteome Array technology is capable of identifying the receptors through which viruses infect cells.

\section{CEL-SCI}

CEL-SCI is developing immunotherapy against COVID-19 using its proprietary LEAPS peptide technology, which utilises conserved areas of the coronavirus proteins to generate $\mathrm{T}$-cell responses and reduce viral load. The technology can also be used to develop immunotherapeutic peptides with both antiviral and anti-inflammatory properties.

The peptides developed using this technology can help in reducing tissue damage from inflammation caused due to lung infection, which is a major cause of mortality in elderly patients.

\section{AJ Vaccines}

AJ Vaccines has launched the development of a vaccine against COVID-19. The company will use the latest technology to develop antigens that can mimic the native structures of the virus. The vaccine will be capable of inducing a strong immune response in the body thereby protecting against the infection.

\section{Takeda Pharmaceutical Company}

Takeda Pharmaceutical Company has announced plans to develop a plasma-derived therapy against coronavirus. The anti-SARS-CoV-2 polyclonal hyperimmune globulin (H-IG) therapy will be designed to treat high-risk patients. The H-IG therapy includes concentrated pathogen-specific antibodies derived from plasma of recovered patients. These antibodies have the potential to generate an immune response when injected into a new patient.

\section{Heat Biologics}

Heat Biologics has announced plans to develop a vaccine to treat or prevent coronavirus infection using its proprietary gp96 vaccine platform. The technology is 
capable of reprogramming live cells to produce antigens that can bind to the gp96 protein and generate an immune response against those antigens.

\section{Pfizer}

Pfizer announced that it has identified certain under development antiviral compounds that may be effective in treating coronavirus. The company is planning to partner with a third party to screen and identify potential compounds by the end of March and begin testing in April.

\section{Mateon Therapeutics}

Mateon Therapeutics has launched an antiviral response programme to develop coronavirus treatments using its therapeutic and artificial intelligence (AI) platforms. It has also established a division, which will adopt a multi-modal approach to developing COVID-19 treatments as well as other future zootonic outbreaks.

\section{Hong Kong University of Science and Technology}

The Hong Kong University of Science and Technology has identified several vaccine targets, which can be developed as a treatment for coronavirus. Researchers at the university have identified B-cell and T-cell epitopes, which are capable of generating an immune response against the SARS virus and a similar response against the coronavirus.

Some of the epitopes identified may be capable of generating an immune response specifically against COVID-19.

\section{Vaccine by Generex}

Generex has announced that it is developing a COVID-19 vaccine following a contract from a Chinese consortium comprising of China Technology Exchange, Beijing Zhonghua Investment Fund Management, Biology Institute of Shandong Academy of Sciences and Sinotek-Advocates International Industry Development.

The company will utilise its Ii-Key immune system activation technology to produce a COVID-19 peptide for human clinical trials.

Generex will receive an upfront payment of $\$ 1 \mathrm{~m}$ to commence the groundwork for the vaccine development and $\$ 5 \mathrm{~m}$ licensing fee for its Ii-Key technology. It is also eligible to receive a $20 \%$ royalty on every dose of vaccine produced under the contract.

\section{Coronavirus drugs by Columbia University}

Researchers at Columbia University have been awarded a $\$ 2.1 \mathrm{~m}$ grant by the Jack Ma Foundation to develop a coronavirus cure. Four different teams at the university will adopt various approaches towards the development of a vaccine against coronavirus.

\section{Vaccine by Tulane University}

Tulane University has launched a research programme to identify a potential medicine for coronavirus in the form of a vaccine. The university will utilise a grant from the Brown Foundation to carry out the research activities.

\section{Coronavirus vaccine by ImmunoPrecise Antibodies}

Immuno Precise Antibodies has launched a vaccine and therapeutic antibody programme to develop a vaccine as well as antibodies against COVID-19. The company will use its B Cell Select ${ }^{\mathrm{TM}}$ and Deep Display $^{\mathrm{TM}}$ discovery platforms to therapeutic compounds against the coronavirus.

The company has updated its research efforts and noted that it will be using the PolyTope mAb Therapy $^{\mathrm{TM}}$ and EVQLV's artificial intelligence platforms develop a COVID-19 therapy.

\section{Serum Institute of India}

Serum Institute of India (SII) is collaborating with Codagenix, a US-based biopharmaceutical company, to develop a coronavirus cure using a vaccine strain similar to the original virus. The vaccine is currently in the pre-clinical testing phase, while human trials are expected to commence in the next six months. SII is expected to launch the vaccine in the market by early 2022.

\section{Southwest Research Institute}

Southwest Research Institute is using its virtual screening called Rhodium to identify potential drug candidates for treating coronavirus from more than two million drug compounds. The most promising compounds will be identified for further development.

\section{Zydus Cadila}

Zydus Cadila announced the launch of an accelerated research programme to develop a vaccine for COVID-19 using two novel approaches. The first approach includes the development of a DNA vaccine against the viral membrane protein of the virus, while a live attenuated recombinant measles virus (rMV) vectored vaccine will be developed in the second approach. The rMV-based vaccine works by inducing specific neutralising antibodies, which will provide protection from the coronavirus infection.

\section{NanoViricides \\ NanoViricides, a clinical-stage company, is working on developing a treatment for nCoV-2019 using its nanoviricide ${ }^{\circledR}$ technology. The company's technology is used to develop ligands that can bind to the virus in the same way as a cognate receptor and attack various points of the virus.}

\section{Vir Biotechnology \\ Vir Biotechnology, a clinical-stage immunology company, announced on 12 February that}


it has identified two monoclonal antibodies that can bind to the virus that causes COVID-19. The antibodies target the spike (S) protein of the virus by entering through the cellular receptor ACE2.

The company has formed a partnership with WuXi Biologics on 25 February to commercialise the antibodies identified to treat coronavirus. If approved, Wuxi will have the rights to market the therapies in China, while Vir will retain the marketing rights in other countries.

Vir has also partnered with Alnylam Pharmaceuticals to identify siRNA candidates targeting SARS-CoV-2. It has formed another partnership with Biogen for cell line and process development and manufacturing of the antibodies.

\section{HIV drugs for coronavirus treatment}

Abbvie's HIV protease inhibitor, lopinavir is being studied along with ritonavir for the treatment of MERS and SARS coronaviruses. The repurposed drug is already approved for the treatment of HIV infection under the trade name Kaletra.

The combination is listed in the WHO list of essential medicines. Lopinavir is believed to act on the intracellular processes of coronavirus replication and demonstrated reduced mortality in the non-human primates (NHP) model of the MERS.

Lopinavir/ritonavir in combination with ribavirin showed reduced fatality rate and milder disease course during an open clinical trial in patients in the 2003 SARS outbreak.

Cipla is also reportedly planning to repurpose its HIV drug LOPIMUNE, which is a combination of protease inhibitors Lopinavir and Ritonavir, for the treatment of coronavirus.

A licensed generic of Kaletra, LOPIMUNE is currently available in packs of 60 tablets each, containing 200mg of Lopinavir and 50mg of Ritonavir.

Janssen Pharmaceutical Companies, a subsidiary of Johnson \& Johnson, donated its PREZCOBIX ${ }^{\circledR}$ HIV medication (darunavir/cobicistat) for use in research activities aimed at finding a treatment for COVID-19.

Darunavir is a protease inhibitor marketed by Janssen. Anecdotal reports suggest darunavir as potentially having antiviral activity against COVID-19. It is, however, currently approved only for use with a boosting agent, and in combination with other antiretrovirals, for the treatment of HIV -1 .
Janssen has no in vitro or clinical data to support the use of darunavir as a treatment for COVID19. The drug is in the process of being evaluated in vitro for any potential activity against the coronavirus.

Further, Janssen has partnered with the Biomedical Advanced Research and Development Authority (BARDA) to expedite the development of a COVID-19 treatment.

\section{The Coronavirus outbreak}

The contagious coronavirus outbreak at the end of 2019, which the WHO named as COVID-19 (formerly 2019-nCoV), led to a medical emergency across the world.

Similar infections are caused by human alpha and beta coronaviruses such as 229E, NL63, OC43 and HKU1.Coronavirus symptoms include those associated with common cold, such as running nose, headache, cough, fever, and sore throat.

\section{Coronavirus transmission}

"Coronavirus transmission can happen humanto-human as well as from infected animals such as dogs and cats."COVID-19 is a beta coronavirus that has origin in bats, according to the Centers for Disease Control and Prevention (CDC).

Believed to have transmitted from animals and reptiles such as snakes, coronaviruses cause respiratory issues such as upper respiratory tract illnesses and lower respiratory illnesses such as pneumonia and bronchitis. Coronavirus transmission can happen human-to-human as well as from infected animals such as dogs and cats.

During public health emergencies declared under section 564 of the FD\&C Act, the FDA is able to issue an Emergency Use Authorizations (EUAs) when certain criteria are met that allows for the use and distribution of potentially life-saving medical products to diagnose, treat, or prevent the disease, which can include diagnostic tests. Accordingly, the FDA has issued EUAs authorizing the use of diagnostic tests to detect the virus, called SARS-CoV-2, that causes COVID-19. The FDA has also issued new policies and guidance to achieve more rapid testing capacity in the U.S.

Because the virus that causes COVID-19 is new, currently there is no FDA-approved or cleared test to diagnose or detect COVID-19.

\section{STATESTICS}

Coronavirus: In India14-hour 'Janta curfew' will not break the cycle of infection and it is extended for another 21 days to avoid further transmission of this COVID-19. 
As of $27 / 3 / 2020$ active covid- 19 is 384,174 out of 531630 in which24,065 ie.,4.53\% death and $123,391(23.21 \%)$ recoveries from this covid-19.India 727 total cases ,662 active covid-19,20 dead and 45 recovered in china 81,285 total cases 3,947 active and3,287 dead and74,051 recovered

\section{CONCLUSION}

From this we can conclude that if isolation and self-quarantine is the better way to prevent further transmission and if this continues for another two weeks, we can successfully eradicate this deadly virus. There is more chance for the recovery from this noxious virus but transmission is very fast through social contact so to avoid this panic situation each and every one should take self-measures to see another dawn.

\section{REFERENCES}

1. Novel Coronavirus (2019-nCoV) Situation Report7 - World Health Organization (WHO).2020; (January 27)

2. China's National Health Commission news conference on coronavirus - Al Jazeera. 2020(26).

3. Symptoms of Novel Coronavirus (2019-nCoV) CDC

4. Novel coronavirus (2019-nCoV) - Australian Government Department of Health

5. Transmission of 2019-nCoV Infection from an Asymptomatic Contact in Germany - The New England Journal of Medicine;2020 (Jan. 30).

6. 'There's no doubt': Top US infectious disease doctor says Wuhan coronavirus can spread even when people have no symptoms - CNN, 2020; (Jan. $31)$.

7. Li Q, Guan X, Wu P, Wang X, Zhou L, Tong Y, Ren R, Leung KS, Lau EH, Wong JY, Xing X. Early transmission dynamics in Wuhan, China, of novel coronavirus-infected pneumonia. New England Journal of Medicine. 2020 Jan 29.

8. Asymptomatic \#2019nCoV infection - WHO Tweet, Feb. 1, 2020

9. Philippines reports coronavirus death, as China toll reaches 304 - Al Jazeera, Feb. 2, 2020

10. Backer Jantien A, Klinkenberg Don, Wallinga Jacco. Incubation period of 2019 novel coronavirus (2019-nCoV) infections among travelers from Wuhan, China, 20-28 January 2020. Euro Surveill. 2020;25(5):

pii=2000062. https://doi.org/10.2807/15607917.ES.2020.25.5.2000062

11. Guan WJ, Ni ZY, Hu Y, Liang WH, Ou CQ, He JX, Liu L, Shan H, Lei CL, Hui DS, Du B. Clinical characteristics of 2019 novel coronavirus infection in China. MedRxiv. 2020 Jan 1.

12. Coronavirus incubation could be as long as 27 days, Chinese provincial government says Reuters. 22; 2020.

13. Bai Y, Yao L, Wei T, Tian F, Jin DY, Chen L, Wang M. Presumed asymptomatic carrier transmission of COVID-19. Jama. 2020 Feb 21.
14. World Health Organization. Clinical management of severe acute respiratory infection when novel coronavirus ( 2019-nCoV) infection is suspected: interim guidance, 28 January 2020. World Health Organization; 2020.

15. Scott, Dylan (16 March 2020). "Coronavirus is exposing all of the weaknesses in the US health system High health care costs and low medical capacity made the US uniquely vulnerable to the coronavirus". Vox. Retrieved 18 March 2020.

16. 3D Printing Media Network. 14 March; 2020. Retrieved 20 March2020.

17. Peters, Jay (17 March 2020). "Volunteers produce 3D-printed valves for life-saving coronavirus treatments". The Verge. Retrieved 20 March 2020.

18. Li G, De Clercq E. "Therapeutic options for the 2019 novel coronavirus (2019-nCoV)". Nature Reviews. Drug Discovery. 2020;19 (3): 149150. doi:10.1038/d41573-020-000160. PMID 32127666.

19. "Chinese doctors using plasma therapy on coronavirus, WHO says 'very valid' approach". Reuters. 17 February 2020 - via www.reuters.com.

20. Steenhuysen J, Kelland K (24 January 2020). "With Wuhan virus genetic code in hand, scientists begin work on a vaccine". Reuters. Archived from the original on 25 January 2020. Retrieved 25 January 2020.

21. Duddu P (19 February 2020). "Coronavirus outbreak: Vaccines/drugs in the pipeline for Covid19". Clinicaltrialsarena.com. Archived from the original on 19 February 2020.

22. Lu H (28 January 2020). "Drug treatment options for the 2019-new coronavirus (2019nCoV)". Biosci Trends. 14(1): 6971. doi:10.5582/bst.2020.01020. PMID 31996494.

23. Nebehay S, Kelland K, Liu R (5 February 2020). "WHO: 'no known effective' treatments for new coronavirus". Thomson Reuters. Archived from the original on 5 February 2020. Retrieved 5 February 2020.

24. "China launches coronavirus 'close contact' app". BBC News. 11 February 2020. Retrieved 7 March 2020.

25. Chen A. "China's coronavirus app could have unintended consequences". MIT Technology Review. Retrieved 7 March 2020.

26. "Gov in the Time of Corona". Gov Insider. 19 March 2020. Retrieved 20 March 2020.

27. Manancourt, Vincent (10 March 2020). "Coronavirus tests Europe's resolve on privacy". POLITICO. Retrieved 20 March 2020.

28. Tidy, Joe (17 March 2020). "Coronavirus: Israel enables emergency spy powers". BBC News. Retrieved 18 March 2020.

29. Paksoy, Yunus. "German telecom giant shares private data with government amid privacy fears". trtworld. Retrieved 20 March 2020. 
30. "Moscow deploys facial recognition technology for coronavirus quarantine". Reuters. 21 February 2020. Retrieved 20 March 2020.

31. "Italians scolded for flouting lockdown as death toll nears 3,000". Pittsburgh Post-Gazette. Retrieved 20 March 2020.

32. Xiang YT, Yang Y, Li W, Zhang L, Zhang Q, Cheung $\mathrm{T}, \mathrm{Ng} \mathrm{CH}$. Timely mental health care for the 2019 novel coronavirus outbreak is urgently needed. The Lancet Psychiatry. 2020 Mar $1 ; 7(3): 228-9$.

33. Kang L, Li Y, Hu S, Chen M, Yang C, Yang BX, Wang Y, Hu J, Lai J, Ma X, Chen J. The mental health of medical workers in Wuhan, China dealing with the 2019 novel coronavirus. The Lancet Psychiatry. 2020 Mar 1;7(3):e14.

34. Roser M, Ritchie H, Ortiz-Ospina E. Coronavirus Disease (COVID-19)-Statistics and Research. Our World in Data. 2020 Mar 4.

35. Novel CP. The epidemiological characteristics of an outbreak of 2019 novel coronavirus diseases (COVID-19) in China. Zhonghua liu xing bing xue za zhi= Zhonghua liuxingbingxue zazhi. $2020 \mathrm{Feb}$ $17 ; 41(2): 145$.

36. "Report of the WHO-China Joint Mission on Coronavirus Disease 2019 (COVID-19)"(PDF). who.int. 16-24 February 2020.

37. Dong Y, Mo X, Hu Y, Qi X, Jiang F, Jiang Z, Tong S. Epidemiological characteristics of 2143 pediatric patients with 2019 coronavirus disease in China. Pediatrics. 2020 Mar 1.
38. Fang L, Karakiulakis G, Roth M. Are patients with hypertension and diabetes mellitus at increased risk for COVID-19 infection?. The Lancet Respiratory Medicine. 2020 Mar 11.

39. "Coronavirus Disease 2019 (COVID-19)". Centers for Disease Control and Prevention. 11 February 2020. Retrieved 2 March 2020.

40. Heymann DL, Shindo N. COVID-19: what is next for public health?. The Lancet. 2020 Feb 22;395(10224):542-5.

41. Cascella M, Rajnik M, Cuomo A, Dulebohn SC, Di Napoli R. Features, Evaluation and Treatment Coronavirus (COVID-19). InStatPearls [Internet] 2020 Mar 8. StatPearls Publishing.

42. Zhou F, Yu T, Du R, Fan G, Liu Y, Liu Z, Xiang J, Wang Y, Song B, Gu X, Guan L. Clinical course and risk factors for mortality of adult inpatients with COVID-19 in Wuhan, China: a retrospective cohort study. The Lancet. 2020 Mar 11.

43. Xu L, Liu J, Lu M, Yang D, Zheng X. Liver injury during highly pathogenic human coronavirus infections. Liver International. 2020 Mar 14.

44. "WHO Director-General's statement on the advice of the IHR Emergency Committee on Novel Coronavirus". World Health Organization (WHO).

45. "Report sulle caratteristiche dei pazienti deceduti positivi a COVID-19 in Italia"(PDF) (in Italian). Rome: Istituto Superiore di Sanità. 17 March 2020.

46. "Epidemia COVID-19. Aggiornamento nazionale 19 marzo 2020"] Check |url=value (help) (in Italian). Rome: Istituto Superiore di Sanità. 19 March 2020. Retrieved 18 March 2020. 